\section{Time and memory}

IgM $^{+}$memory B cells exist in mice; however, their contribution to the antigen-specific memory pool has remained unclear because of technical difficulties in detecting rare endogenous antigen-specific cells. In Science, Jenkins and colleagues use an antigen-based technique for the enrichment of rare cells to show that immunization with antigen generates both immunoglobulinswitched and $\operatorname{lgM}^{+}$memory B cells. IgM+ cells, which show little evidence of selection in the germinal center or affinity maturation, are more numerous and have a longer lifespan than immunoglobulin-switched cells but expand their populations poorly during a secondary response. In contrast, immunoglobulinswitched populations expand greatly and dominate the secondary response because of their ability to be activated in the presence of high-affinity serum immunoglobulin. Thus, early memory relies on shorter-lived immunoglobulin-switched cells, whereas more durable $\mathrm{IgM}^{+}$reserves account for late memory.

Science (10 February 2011) doi:10.1126/science.1201730

\section{Location matters}

Medullary thymic epithelial cells (mTECs) that express the transcriptional regulator Aire contribute to central tolerance mechanisms, but whether these cells likewise influence the generation of natural regulatory $\mathrm{T}$ cells $\left(\mathrm{nT}_{\mathrm{reg}}\right.$ cells) is not clear. In the Journal of Experimental Medicine, Lei et al. show that mTECs express the chemokine XCL1 (lymphotactin) to recruit dendritic cells (DCs) positive for its receptor XCR1 to the thymic medulla, where these cells act together to produce $\mathrm{nT}_{\text {reg }}$ cells. Aire is required for Xcl1 expression. Although mice deficient in Xcl1 undergo normal negative selection of thymocytes, they have defective generation of $\mathrm{nT}_{\text {reg }}$ cells and develop age-related autoimmune manifestations. Thymic DCs accumulate at corticomedullary junctions in mice that lack XCL1, which suggests that proximity of thymic DCs with mTECs is necessary for the proper development or selection of $\mathrm{nT}_{\text {reg }}$ populations.

$L A D$

J. Exp. Med. 208, 383-394 (2011)

\section{Obesity, inflammation and NLRP3}

Obesity is characterized by chronic low-grade inflammation, but how this state is engendered in the absence of any overt infection remains unclear. In Nature Medicine, Dixit and colleagues show that the cytosolic pattern-recognition complex NLRP3 is fundamental to obesity-induced inflammation and associated complications such as insulin resistance. NLRP3 is triggered by a variety of intrinsic and extrinsic signals; this ultimately results in the release of proinflammatory cytokines such as IL-1 $\beta$ and IL-18. Dixit et al. find that the expression of NIrp3 and $/ / 1 b$ is higher in the fatty tissues of obese mice and humans. NLRP3 is associated mainly with fatty tissue-infiltrating macrophages that, furthermore, drive local $\mathrm{T}$ cells to an inflammatory $\mathrm{T}$ helper type 1 phenotype through their release of IL-18. Obese mice have impaired insulin sensitivity that is normalized by ablation of NIrp3. Free fatty acid-derived ceramide that results from a highfat diet activates NIrp3 in macrophages in vitro. NLRP3 is thus a culprit in obesity-associated inflammation.

Nat. Med. 17, 179-188 (2011)

\section{Recovering responses with IL-7}

The immunological processes that lead to chronic viral infection are relatively well understood, but effective therapies to resolve such infections have remained elusive. In Cell, Mak and colleagues identify a role for the homeostatic cytokine IL-7 in normalizing the 'exhausted' immune response of chronic infection. Using the lymphocytic choriomeningitis virus clone 13 mouse model of persistent viral infection, the authors find that IL-7 administration effectively clears viral infection. IL-7 seems to exert its effects at multiple levels. Not only does IL-7 boost the proliferation and effector function of virus-specific $\mathrm{T}$ cells, it also seems to reverse the exhausted $\mathrm{T}$ cell phenotype normally seen in chronic infection. Mechanistically, IL-7 represses T cell expression of SOCS3 - an inhibitor of inflammatory cytokine signaling that is thus broadly antagonistic to antiviral responses-without triggering pathogenic inflammation. IL-7 is therefore a promising candidate for the treatment of persistent infection with virus, including human immunodeficiency virus.

Cell 144, 601-613 (2011)

\section{Anergy control}

Although anergic, self-reactive T cells can persist for long periods of time, the molecular mechanisms that control T cell anergy remain poorly understood. In Immunity, Abbas and colleagues show that a cytokine-specific block in mRNA translation occurs in anergic T cells. Although TCR-driven transcription of genes encoding lineage-restricted cytokines such as interferon- $\gamma$, IL-4, IL-13, IL-17A, IL-17F and IL-21 is induced in T cells responding to self or foreign antigen, anergic $\mathrm{T}$ cells cannot generate cytokine proteins. The mRNA for these cytokines contains conserved AU-rich elements in the $\mathbf{3}^{\prime}$ untranslated region. This AU-rich element-dependent mechanism prevents cytokine translation in a tolerogenic setting but not in a lymphopenic or autoimmune setting. How post-transcriptional silencing is inhibited or overcome when $\mathrm{T}$ cells are primed under inflammatory conditions is not clear.

Immunity 34, 50-60 (2011)

\section{Regulating inflammatory genes}

The expression of genes encoding inflammatory molecules is subject to multiple layers of regulation. In Nature, Glass and colleagues identify a regulatory network that governs the expression of genes suppressed by nuclear corepressor (NCoR) complexes. NCoR prevents expression of Nos 2 and Ccl12 by binding to their promoters. The nuclear actin-binding factor coronin $2 \mathrm{~A}$ relieves $\mathrm{NCoR}$ repression by promoting chromatin remodeling of these regulatory sites. However, liver X receptors (LXRs) that undergo ligand-dependent SUMOylation block this de-repression. Coronin 2A contains a SUMO-interaction domain that recognizes the activated LXRs and prevents its ability to remove the repressive NCoR complexes. Inflammatory stimuli such as TLR ligation trigger phosphorylation of LXRs and loss of their SUMO modification, thereby relieving inhibition of coronin 2A activity. In Science Signaling, Cheng et al. identify a subset of interferon-inducible genes that contain guanine-rich interferon-response elements that are also recognized and repressed by homodimers of the transcription factor NF- $\kappa$ B subunit $\mathrm{p} 50$. Such regulation allows stimulus-appropriate activation of genes regulated by NF- $\mathrm{B}$ and IRF transcription factors.

$L A D$ Nature 470, 414-418 (2011); Sci. Signal. (22 February 2011) doi:10.1126/ scisignal.2001501 